\title{
The role of intra-arterial chemotherapy in retinoblastoma treatment
}

\author{
Reed Jenkins and Efren González* \\ Department of Ophthalmology, Boston Children's Hospital, Boston, Massachusetts, United States of America
}

\begin{abstract}
Intra-arterial chemotherapy (IAC) has recently emerged as an important treatment modality in the management of retinoblastoma. In this review, we detail the development of IAC, its efficacy, associated adverse effects, and its expanding role in treating different presentations of retinoblastoma.
\end{abstract}

Key words: Retinoblastoma. Drug therapy. Chemotherapy. Cancer. Regional perfusion. Adverse effects.

\section{Introduction}

Retinoblastoma is the most prevalent intraocular malignancy in the pediatric population, comprising between 2.5 and $4 \%$ of all childhood neoplasms ${ }^{1}$. Kivelä estimated that 7202 to 8102 cases of retinoblastoma will develop annually, based on a worldwide incidence of $1: 16,000$ to $1: 18,000$ live births ${ }^{2}$.

Retinoblastoma can involve either one or both eyes; approximately $40 \%$ of cases are bilateral, and $60 \%$ are unilateral. Bilateral retinoblastoma is heritable, with children having a mutation in one of the alleles of the RB1 gene. Unilateral cases are non-familial, with mutations occurring sporadically ${ }^{3}$. Beyond the genetic differences, unilateral and bilateral retinoblastoma also diverge in the age at which children present. The mean age at which children present with bilateral disease is around 12 months, while the mean age at diagnosis for unilateral disease is 24 months ${ }^{4}$. Children with retinoblastoma most often present clinically in the developed world with a white reflex in the eye, leukocoria, or strabismus caused by tumor involvement in the macular region ${ }^{1,5,6}$. In a large, retrospective review of retinoblastoma patients, Abramson et al. found that leukocoria and strabismus were the most common presenting signs for both unilateral and bilateral cases ${ }^{5}$.

There are several different staging and classification systems utilized to characterize retinoblastoma upon diagnosis. One of the most commonly used systems is the International Intraocular Retinoblastoma Classification (IIRC), wich was developed in the 1990s and groups intraocular tumors from A-E. Tumors are classified on several characteristics, including their size and location, with Group E retinoblastoma the most severe ${ }^{7}$.

Group A: Small tumors ( $<3 \mathrm{~mm}$ or smaller) away from foveola and optic disc

Group B: All remaining tumors confined to the retina

Group C: Discrete retinal tumors with focal vitreous or subretinal seeding

Group D: Diffuse retinal or vitreous seeding

Date of reception: $13-10-2020$

Date of acceptance: 14-10-2020

E-mail: efren.gonzalez@childrens.harvard.edu DOI: 10.24875/RMU.M20000053

1665-5796/С 2020 Universidad Autónoma de Nuevc (http://creativecommons.org/licenses/by-nc-nd/4.0/)

Available online: $24-12-2020$ Medicina Universitaria. 2020;22(4):188-192 www.medicinauniversitaria.org . Published by Permanyer. This is an open access article under the CC BY-NC-ND license 
Group E: Very high-risk eyes destroyed anatomically or functionally with one or more of the following: neovascular hemorrhage, massive intraocular hemorrhage, aseptic orbital cellulitis, tumor touching the lens, diffuse infiltrating retinoblastoma, and phthisis or pre-phthisis?

Since the first report of an intraocular tumor resembling retinoblastoma by Petrus Pawius of Amsterdam in 1657 , scientific understanding of retinoblastoma has proceeded in leaps and bounds, enabling the discovery of new techniques and therapies to treat the disease ${ }^{8}$. In 1971, Knudson developed a theory on the genetic etiology of retinoblastoma with his "two-hit hypothesis $^{9,10}$." Knudson postulated that retinoblastoma occurs after two "hits" or mutations. In the sporadic form, often manifesting in unilateral cancer, two somatic mutations cause retinoblastoma to develop, usually at a later age. In the heritable form, often resulting in bilateral tumors, one germline mutation is inherited and followed by a somatic mutation ${ }^{9,10}$. Knudson's work helped to elucidate the concept of a "tumor suppressor gene," whose inactivation allows uncontrolled cell division and malignant growth.

In 1986, Friend et al. cloned RB1, the tumor suppressor gene, which leads to the formation of retinoblastoma when inactivated, on the q14 band of chromosome $13^{11}$. This discovery marked a seminal advance in cancer biology and further explained the inheritance patterns of retinoblastoma. While inactivation of RB1 is responsible for the development of the vast majority of retinoblastomas, Rushlow et al. demonstrated that amplification of the MYCN oncogene might alone initiate retinoblastoma ${ }^{3}$. MYCN-amplified retinoblastoma has distinct histological features, is unilateral, and arises at an early age $^{3}$.

Advances in the genetic understanding of retinoblastoma have been accompanied by the emergence of new strategies to manage the disease. With the advent of the ophthalmoscope in the mid-19th century and the detection of retinoblastoma at earlier intraocular stages, enucleation became the first widely-accepted treatment for the disease ${ }^{8}$. In the early $20^{\text {th }}$ century, ophthalmologists began to use radiation to treat retinoblastoma, especially in bilateral disease after the enucleation of the more affected eye ${ }^{8}$. However, the serious side effects of radiation treatment, including ocular toxicity, led to a refinement of radiation dosage and techniques ${ }^{12}$. Even with these refinements, Wong et al. demonstrated that radiotherapy increased the risk of secondary malignancies in patients with hereditary retinoblastoma. The rate of sarcoma development vastly exceeded that of the general population ${ }^{13}$. Due to this risk and accompanying treatment site side effects, including cataracts and craniofacial deformities, centers began to move away from radiation therapy ${ }^{14}$.

In the 1990s, the paradigm of retinoblastoma management shifted to an approach characterized by the use of chemotherapy and local consolidation therapies such as cryotherapy and photocoagulation to manage most bilateral and some unilateral retinoblastomas ${ }^{12,14,15}$. Shields et al. established the efficacy of chemoreduction along with adjuvant therapies in controlling intraocular retinoblastoma ${ }^{15}$. However, systemic chemotherapy, such as radiation therapy, can cause significant adverse effects in children, including hearing impairment, possible impacts on fertility, and a link between chemotherapy and the development of secondary acute myelogenous leukemia (SAML) later in life ${ }^{16}$. In the short-term, many patients face fever, neutropenia, red blood cell transfusions, and the need for port installation ${ }^{16}$. While enucleation, chemotherapy, and other classic retinoblastoma treatments remain important tools, the desire to find effective therapies with less systemic and ocular toxicity led to the development of intra-arterial chemotherapy to treat retinoblastoma ${ }^{12}$.

\section{Development and technique of IAC for retinoblastoma}

Although the technique of intra-arterial delivery of chemotherapy (IAC) to treat retinoblastoma was first reported over 50 years ago, it has only become widely accepted in the last 15, as technical advancements have improved tumor control and minimized toxicity. The first report of IAC for retinoblastoma was in 1958 by Reese et al., who injected triethylene melamine (TEM) into the internal carotid artery of 23 patients with advanced retinoblastoma ${ }^{17}$. In 2004, Yamane et al. reported the results of an updated technique for IAC in patients with retinoblastoma ${ }^{18}$. The new technique used a balloon catheter to occlude flow in the internal carotid artery (ICA) distal to the ophthalmic artery's ostium. In this fashion, chemotherapeutic agents could be infused more selectively into the ophthalmic region ${ }^{14,18}$. Yamane et al. treated 187 patients for 563 sessions of IAC, with a technical success rate of $97.5 \%$ and no significant complications ${ }^{18}$. However, the balloon occlusion method poses two significant risks to pediatric patients: arterial dissection and thrombosis within the ICA, leading to embolization into the cranial circulation ${ }^{14}$. The positive findings of Yamane et al. and the promise they held for the management of intraocular retinoblastoma spurred the refinement of "super-selective" ophthalmic 
artery infusion by a team in New York led by David Abramson and Pierre Gobin, among others ${ }^{16}$.

The refined technique pioneered by Abramson et al. draws on interventional radiologic techniques to selectively cannulate the ophthalmic artery ${ }^{19}$. In this approach, general anesthesia is administered. The femoral artery is punctured, a 4-French $(F)$ sheath is inserted, and anticoagulation is administered. A catheter is maneuvered to the ipsilateral internal carotid artery, and an arteriogram is performed to visualize the cerebral and ocular vasculature. Then, the ophthalmic artery is selectively catheterized using a microcatheter. Once the catheter is stable in the ostium of the ophthalmic artery, an angiogram is used to confirm the position, and the chemotherapeutic agent is infused in a pulsatile fashion. After the infusion, the catheter is retracted, the femoral sheath is withdrawn, and manual compression is applied to attain hemostasis. Anesthesia is reversed, the patient is monitored for 4-6 $\mathrm{h}$ in the PACU, and discharged the same day ${ }^{14,16,19,20}$.

\section{Efficacy of IAC}

In 2008, Abramson et al. published results from an initial Phase I/II study of superselective ophthalmic chemosurgery in a group of 9 advanced eyes with retinoblastoma scheduled for enucleation. Seven of the nine eyes avoided enucleation with minimal systemic and ocular toxicity. Qualitatively, the tumor and vitreous seeding response to IAC was often "dramatic"19. In the same cohort of patients, it was shown that retinal function could endure through IAC and even improve, with three treated patients recording improved responses on $E R G^{21}$. The initial results of IAC in New York were more effective at salvaging eyes with advanced intraocular retinoblastoma than the traditional methods of intravenous chemoreduction and external beam radiotherapy ${ }^{22}$. The positive results were replicated by Shields et al. in Philadelphia, who demonstrated the efficacy of IAC as both a first-line and salvage treatment for refractory retinoblastoma. In a retrospective review of 36 eyes with IAC as a primary treatment and 34 eyes with IAC as a secondary treatment, complete regression of tumor was achieved in $94 \%$ of eyes and resolution of subretinal seeds in $95 \%$ of eyes. IAC improved globe salvage in patients with advanced (Group D and E) intraocular retinoblastoma ${ }^{23}$. IAC has since become one of the frontline management strategies for retinoblastoma, especially in treating advanced intraocular retinoblastoma, where it has shown a pronounced effect. This has been apparent in treating advanced Group D eyes, leading to higher rates of globe salvage and new options for patients and families facing advanced disease ${ }^{24}$.

\section{Complications of IAC}

Although IAC has opened new treatment options for patients with retinoblastoma, there are still questions about its risks and potential complications. Because IAC does not deliver systemic protection like intravenous chemotherapy, there have been concerns about the potential risk for metastasis in advanced retinoblastoma cases. However, there have been few cases of metastases in IAC treatment cohorts ${ }^{24}$. A retrospective review on outcomes after IAC was conducted by six international retinoblastoma centers and found that of 1139 patients treated over 10 years, there were only three metastatic deaths, and all from one center in Buenos Aires ${ }^{25}$.

Despite the focal delivery of chemotherapy in IAC, some patients still experience toxicity from the chemotherapeutic dosage. In 2011, Gobin et al. found significant neutropenia after $11.4 \%$ of 255 treatment sessions. Neutropenia was either Grade 3 or Grade 4, and one patient was admitted for antibiotics with a fever ${ }^{22}$. In a meta-analysis of IAC outcomes and complications, Ravindran et al. found that neutropenia (16.5\%), fever $(16.2 \%)$, and nausea (11.6\%) were relatively common side effects in patients. However, there were no reports of patients needing transfusions for neutropenia ${ }^{26}$.

There have also been reports of patients suffering neurologic complications from the procedure, including a post-operative seizure caused by a small cerebral infarct and a patient who suffered a stroke resolved with antiplatelet therapy. Both patients recovered without neurologic sequelae ${ }^{27,28}$. There have also been reports of patients suffering anaphylactic reactions during the procedure, potentially in response to the chemotherapeutic agents administered ${ }^{29,30}$. There have also been few reports of puncture site complications. In their 4-year report on IAC, Gobin et al. described one case of transient occlusion of the femoral artery recanalized with aspirin after one week ${ }^{22}$. Hemorrhage and groin hematoma have also been reported $^{31,32}$. A final vascular complication reported in children undergoing IAC is Blue Toe Syndrome. In one case report, a 7-month-old treated with three cycles of IAC returned for a next-day follow-up with a cyanotic and tender toe. After treatment with anticoagulation and aspirin, the symptoms resolved within 10 days $^{28,33}$. However, some of these vascular adverse effects can likely be mitigated by experience and careful angiographic technique for infants ${ }^{14}$. 
Another important consideration when administering IAC is the widespread reports of serious hemodynamic instability and bronchospasm during procedures. In 2011, Gobin et al. reported 24 cases of bronchospasm, all occurring in the second session of IAC or later. In these instances, bronchospasm occurred when the microcatheter reached the carotid or ophthalmic arteries and sometimes led to bradycardia and hypotension. When a bronchospasm occurred, the catheterization was halted, and epinephrine was injected. All patients recovered and were successfully cannulated ${ }^{22}$. Phillips et al. reported adverse cardiorespiratory events in $24 \%$ of procedures, with one IAC session unsuccessful due to prolonged hemodynamic instability ${ }^{34}$. In 2015 , Kato et al. published a review of anesthesia records for patients undergoing IAC at a major center and found that $67 \%(63 / 94)$ of patients who underwent IAC had a severe respiratory compliance event during at least one procedure. All events were transient and did not cause significant morbidity or admission ${ }^{35}$. In a prospective study, Nghe et al. found a $20 \%$ incidence of severe cardiorespiratory events during IAC, with the vast majority (17\%), occurring within 2 min of situating the catheter in the ostium of the ophthalmic artery ${ }^{36}$. These compliance changes are likely due to an unspecified autonomic reflex ${ }^{35,36}$. The findings emphasize the necessity of vigilance during IAC and the importance of educating families on the procedure's associated risks.

A final consideration for the use of IAC is the potential for ocular and periocular adverse effects associated with the procedure. In their meta-analysis of studies evaluating the efficacy and complications of IAC, Ravindran et al. found that the most common ocular complications were retinal detachment, retinal or choroidal ischemia, vitreous hemorrhage, and chorioretinal atrophy ${ }^{26}$. In a retrospective histopathological analysis, Biewald et al. found that $10 \%$ of eyes treated with IAC showed serious toxic vascular complications. These vascular events could severely compromise visual potential and may be correlated with cumulative toxicity from chemotherapeutic treatment ${ }^{37}$. There have also been reports of periocular edema and ocularmotor nerve palsy in patients after IAC treatment ${ }^{14,26}$. However, with improvements in knowledge and technique, these complications now occur in less than $5 \%$ of treated eyes ${ }^{38}$.

\section{Conclusion}

Looking toward the future, the goals of retinoblastoma management have not changed. Treatment emphasizes first the preservation of life, followed by the preservation of the eye and usable vision ${ }^{23}$. The development of intra-arterial chemotherapy and its adoption in centers worldwide has transformed our approach to retinoblastoma, along with the increasing use of intravitreal chemotherapy as a complementary therapy. In a discussion between four leading retinoblastoma center leaders on treatment strategies for retinoblastoma, the authors agreed on the prominent role of IAC in treating different scenarios. For unilateral Group D eyes, the authors agreed on IAC as a primary treatment, with a reported globe salvage rate of $94 \%$. For Group E eyes, the authors reported an increasing use of IAC as a primary therapy in eyes that did not show signs requiring immediate enucleation, with increasing salvage of these advanced cases ${ }^{38}$. IAC is also increasingly accepted as a bilateral retinoblastoma treatment, with some centers performing tandem therapy for advanced bilateral cases. These widespread applications have led to a paradigm shift in treatment for retinoblastoma ${ }^{38}$. They have also emphasized the importance of an interdisciplinary approach to retinoblastoma care, with the expertise of a neuro-interventional radiologist or neurosurgeon complemented by the ocular oncologist, pediatric oncologist, anesthesiologist, and other skilled staff members ${ }^{24}$. More studies will be needed to assess the use of IAC in expanded contexts and in different patient populations, and our continued experience will lead to refinement. However, our journey toward curing retinoblastoma and preserving vision has been transformed by the establishment of IAC as an effective and safe treatment.

\section{Conflicts of interest}

The authors declare no conflicts of interest.

\section{Funding}

The authors received no specific funding for this work.

\section{References}

1. Rodriguez-Galindo C, Orbach DB, VanderVeen D. Retinoblastoma. Pediatr Clin North Am. 2015;62:201-23.

2. Kivelä $T$. The epidemiological challenge of the most frequent eye cancer: retinoblastoma, an issue of birth and death. BMJ. 2009;93:1129-31.

3. Rushlow DE, Mol BM, Kennett JY, Yee S, Pajovic S, Thériault BL, et al. Characterisation of retinoblastomas without RB1 mutations: genomic, gene expression, and clinical studies. Lancet Oncol. 2013;14:327-34.

4. Melamud A, Palakar R, Singh A. Retinoblastoma. Am Fam Physician. 2006;73:1039-44.

5. Abramson DH, Frank CM, Susman M, Whalen MP, Dunkel IJ, Boyd NW $3^{\text {rd }}$. Presenting signs of retinoblastoma. J Pediatr. 1998;132:505-8.

6. Dimaras H, Kimani K, Dimba EA, Gronsdahl P, White A, Chan HS, et al. Retinoblastoma. Lancet. 2012;379:1436-46. 
7. Fabian ID, Reddy A, Sagoo MS. Classification and staging of retinoblastoma. Community Eye Health. 2018;31:11-3.

8. Albert DM. Historic review of retinoblastoma. Ophthalmology. 1987;94:654-62.

9. Knudson AG. Mutation and cancer: statistical study of retinoblastoma. Proc Natl Acad Sci. 1971;68:820-3.

10. Knudson AG. Two genetic hits (more or less) to cancer. Nat Rev Cancer. 2001;1:157-62.

11. Friend SH, Bernards R, Rogelj S, Weinberg RA, Rapaport JM, Albert DM, et al. A human DNA segment with properties of the gene that predisposes to retinoblastoma and osteosarcoma. Nature. 1986;323:643-6.

12. Abramson $\mathrm{DH}$. Retinoblastoma in the $20^{\text {th }}$ century: past success and future challenges the Weisenfeld lecture. Investig Ophthalmol Visual Sci. 2005;46:2684-91.

13. Wong FL, Boice JD, Abramson DH, Tarone RE, Kleinerman RA, Stovall $\mathrm{M}$, et al. Cancer incidence after retinoblastoma: radiation dose and sarcoma risk. JAMA. 1997;278:1262-7.

14. Monroy JE, Orbach DB, VanderVeen D. Complications of intra-arterial chemotherapy for retinoblastoma. Semin Ophthalmol 2014;29:429-33.

15. Shields CL, Shields JA, Needle M, de Potter P, Kheterpal S, Hamada A et al. Combined chemoreduction and adjuvant treatment for intraocular retinoblastoma. Ophthalmology. 1997;104:2101-11.

16. Abramson $\mathrm{DH}$. Super selective ophthalmic artery delivery of chemotherapy for intraocular retinoblastoma: chemosurgery the first Stallard lecture. Br J Ophthalmol. 2010;94:396-9.

17. Reese A, Hyman G, Forrest A. The treatment of retinoblastoma by x-ray and triethylene melamine. AMA Arch Ophthalmol. 1958;60:897-906.

18. Yamane $T$, Kaneko A, Mohri M. The technique of ophthalmic arterial infusion therapy for patients with intraocular retinoblastoma. Int J Clin Oncol. 2004;9:69-73.

19. Abramson DH, Dunkel IJ, Brodie SE, Kim JW, Gobin YP. A phase I/I study of direct intraarterial (ophthalmic artery) chemotherapy with melphalan for intraocular retinoblastoma: initial results. Ophthalmology. 2008;115:1398-404.e1.

20. Abramson DH, Dunkel IJ, Brodie SE, Marr B, Gobin YP. Superselective ophthalmic artery chemotherapy as primary treatment for retinoblastoma (chemosurgery). Ophthalmology. 2010;117:1623-9.

21. Brodie SE, Gobin YP, Dunkel IJ, Kim JW, Abramson DH. Persistence of retinal function after selective ophthalmic artery chemotherapy infusion for retinoblastoma. Doc Ophthalmol. 2009;119:13-22.

22. Gobin YP, Dunkel IJ, Marr BP, Brodie SE, Abramson DH. Intra-arterial chemotherapy for the management of retinoblastoma: four-year experience. Arch Ophthalmol. 2011;129:732-7.

23. Shields CL, Manjandavida FP, Lally SE, Pieretti G, Arepalli SA, Caywood $\mathrm{EH}$, et al. Intra-arterial chemotherapy for retinoblastoma in 70 eyes: outcomes based on the international classification of retinoblastoma. Ophthalmology. 2014;121:1453-60.

24. Manjandavida FP, Stathopoulos C, Zhang J, Honavar SG, Shields CL. Intra-arterial chemotherapy in retinoblastoma-a paradigm change. Indian J Ophthalmol. 2019;67:740.
25. Abramson $\mathrm{DH}$, Shields $\mathrm{CL}$, Jabbour $\mathrm{P}$, Teixeira LF, Fonseca JR, Marques MC, et al. Metastatic deaths in retinoblastoma patients treated with intraarterial chemotherapy (ophthalmic artery chemosurgery) worldwide. Int J Retina Vitreous. 2017;3:40.

26. Ravindran K, Dalvin LA, Pulido JS, Brinjikji W. Intra-arterial chemotherapy for retinoblastoma: an updated systematic review and meta-analysis. J Neurointerv Surg. 2019;11:1266-72.

27. Ammanuel S, Alexander MD, Damato B, Cooke DL, Halbach VV, Amans MR, et al. Improved procedural safety following protocol changes for selective ophthalmic arterial infusion of chemotherapy for treatment of ocular retinoblastoma. Interv Neuroradiol. 2018;24:345-50.

28. Oto B, Sarıcı AM, Kızılkılıç O. Superselective intra-arterial chemotherapy treatment for retinoblastoma: clinical experience from a tertiary referral centre. Can J Ophthalmol. 2020;55:406-12.

29. Requejo F, Marelli J, Johnson AR, Sampor C, Chantada G. The technique of superselective ophthalmic artery chemotherapy for retinoblastoma: the Garrahan hospital experience. Interv Neuroradiol. 2018;24:93-9.

30. Tuncer S, Sencer S, Kebudi R, Tanyıldız B, Cebeci Z, Aydın K. Superselective intra-arterial chemotherapy in the primary management of advanced intra-ocular retinoblastoma: first 4-year experience from a single institution in Turkey. Acta Ophthalmol. 2016;94:e644-51.

31. Liu CC, Mohmood A, Hamzah N, Lau JH, Khaliddin N, Rahmat J. Intra-arterial chemotherapy for retinoblastoma: our first three-and-a-half years' experience in Malaysia. PLoS One. 2020;15:e0232249.

32. Radros J, All-Eriksson C, Pal N, Holm S, Seregard S, Söderman M, et al. Intra-arterial chemotherapy for retinoblastoma in Sweden-evaluation of treatment efficacy and complications. Acta Ophthalmol. 2018;96: e1040-1.

33. Sarici A, Kizilkilic O, Celkan T, Gode S. Blue toe syndrome as a complication of intra-arterial chemotherapy for retinoblastoma. JAMA Ophthalmol. 2013;131:801-2.

34. Phillips TJ, McGuirk SP, Chahal HK, Kingston J, Robertson F, Brew S, et al. Autonomic cardio-respiratory reflex reactions and superselective ophthalmic arterial chemotherapy for retinoblastoma. Paediatr Anaesth. 2013;23:940-5.

35. Kato MA, Green N, O'Connell K, Kramer DJ, Al-Khelaifi M, Han HJ, et al. A retrospective analysis of severe intraoperative respiratory compliance changes during ophthalmic arterial chemosurgery for retinoblastoma. Paediatr Anaesth. 2015;25:595-602.

36. Nghe MC, Godier A, Shafii A, Leblanc I, Picard H, Blanc R, et al. Prospective analysis of serious cardiorespiratory events in children during ophthalmic artery chemotherapy for retinoblastoma under a deep standardized anesthesia. Pediatr Anesth. 2017;28:120-6.

37. Biewald EM, Bornfeld N, Metz KA, Schlüter $S$, Kiefer T, Radbruch $A$. et al. Histopathology of retinoblastoma eyes enucleated after intra-arterial chemotherapy. Br J Ophthalmol. 2019;104:1171-5.

38. Abramson DH, Shields CL, Munier FL, Chantada GL. Treatment of retinoblastoma in 2015: agreement and disagreement. JAMA Ophthalmol. 2015;133:1341-7. 